\title{
Effects of Calcium Antagonists on the Risks of Coronary Heart Disease, Cancer and Bleeding
}

\author{
Ad Hoc Subcommittee* of the Liaison Committee of the World Health Organisation \\ and the International Society of Hypertension
}

\begin{abstract}
Background
Following the recent controversy in the medical and lay press about the safety of calcium antagonists, the Liaison Committee of the World Health Organisation and the International Society of Hypertension formed an ad hoc subcommittee to review the relevant available evidence about the effects of these agents on the risks of coronary heart disease (CHD), cancer, and bleeding. In July 1996, the subcommittee sought submissions through notices in medical journals and through direct invitations to the authors of relevant recent research reports and commentaries. Evidence was also sought directly from the published literature through computer searches of the Medline and Current Contents databases and through scrutiny of the reference lists of relevant published papers. This report was drafted after review of the available evidence and was issued by the subcommittee on 10 February, 1997.
\end{abstract}

\section{Scope of the Report}

The primary objectives of this report were to consider systematically the available evidence about the effects of calcium antagonists on the risks of major CHD events [defined here as fatal or non-fatal myocardial infarction, or other death (sudden or not) from CHD] and of cancer (fatal or not). A secondary objective was to review the available evidence about the effects of calcium antagonists on bleeding risks. These represent the main outcomes for which there is controversy about the effects of calcium antagonists. Evidence about the effects of these agents on other less frequent or less serious outcomes that have occasionally been reported to be associated with calcium antagonist use was not considered. Data were reviewed on all types of calcium antagonists, including verapamil, diltiazem and the dihydropyridine drugs, such as nifedipine. Evidence was considered from a variety of sources including systematic overviews (meta-analyses) of randomised trials, individual randomised trials, nonrandomised trials and observational studies. Both published and unpublished evidence was consid- ered. Most of the evidence reviewed was from studies conducted in patients with hypertension (with or without CHD) or in patients with a history of myocardial infarction (with or without hypertension).

\section{Methodological Principles}

In conducting this review, the subcommittee acknowledged that the true effects of calcium antagonists would be determined most reliably from data in which both systematic errors (biases) and random errors (imprecision) were minimised. In this regard, the subcommittee was guided by the following general methodological principles that apply to the assessment of evidence about the effects of any medical intervention.

\section{Minimisation of Systematic Errors}

Systematic errors in estimates of treatment effect are reduced in data from randomised controlled trials as a consequence of the random assignment of individual patients to study treatment and control groups; randomisation minimises the likelihood of there being prognostically important differences between the groups at entry to the study (1). Systematic errors are further reduced by basing estimates of treatment effect on a complete overview of the results of all relevant randomised trials, thereby minimising the possibility that such estimates will reflect results of a non-representative subgroup of trials (e.g. those that may be atypically positive or negative) (2). Data from trials in which the study treatment is allocated in a non-random manner, such as alternate patient allocation, can be affected by substantial systematic errors, particularly when the investigator has foreknowledge of the treatment allocation schedule (1).

The potential for systematic error is greatest, however, in observational studies of treatments selectively provided to individual patients when thought to be specifically indicated by the doctors responsible for their care. In such studies, it is very difficult to distinguish differences in outcome that are the consequence of the indication for treatment from differences in outcome that are conferred by

* Members of the Subcommittee are listed in the Acknowlegements (page 70).

(Hypertens Res 1997; 20: 61-73) 
the treatment itself. For example, some observational studies of patients following myocardial infarction reported increased mortality rates in those treated with digoxin (3), but until recently (4) it was unclear whether this represented a true adverse effect of digoxin or the selection for treatment of higher-risk patients (e.g., those with heart failure or atrial fibrillation) (5). In general observational studies can provide useful evidence about the effects of a treatment only when these are both very large and unlikely to be explained by known or unknown confounding due to indication. An example of such evidence is the demonstration by observational studies of an approximately 7 -fold increase in the risk of endometrial cancer in patients given longterm oestrogen-replacement therapy unopposed by progestogen (6). In the assessment of more moderate effects or of outcomes likely to be substantively affected by confounding, observational studies are less useful, since multivariate statistical adjustment is rarely able to ensure that residual differences in outcome are not due to residual confounding by indication. For example, whereas overviews of randomised controlled trials of both antihypertensive treatment $(7,8)$ and aspirin $(9,10)$ have clearly demonstrated that these treatments produce moderate reductions in the risks of non-fatal myocardial infarction or CHD death, observational studies have reported increased risks of CHD events among patients treated with antihypertensive drugs (11) or aspirin (12), even after adjustment for a variety of confounding factors. These misleading findings from observational studies seem likely to reflect persisting "indication bias" in the observational studies, despite efforts to reduce confounding through multivariate statistical adjustment. The failure of such statistical adjustment is due to both insufficient correction for recorded confounding factors (due to random errors in their measurement) and lack of correction for confounding factors that were not recorded. The consequence of this is that observational studies may provide unreliable information, not just about the size of any treatment effect, but even about its direction.

\section{Minimisation of Random Errors}

In studies of disease risk, random errors are inversely proportional to the number of individuals observed to develop the disease during follow-up. Thus, random errors are smallest, and the precision of estimates of treatment effect is greatest when large numbers of events are observed. Particularly large numbers of events are required for the reliable detection of small-to-moderate treatment effects. For common types of cardiovascular disease and cancer, the most plausible expectation about the effects of any new treatment on major disease events is either no effect, modest harm or modest benefit (1). Therefore determination of the most likely effects of calcium antagonists on the risks of major CHD events or cancer is likely to require studies in which large numbers of outcome events are observed. For example, to detect a plausibly modest increase or decrease in risk of about one- fifth or one-sixth requires data from a study (or an overview of studies) in which at least 1,000 patients develop the relevant disease during follow-up. While very large differences in disease frequency can be detected with many fewer observed cases, effects of such magnitude are unusual (particularly for widely prescribed drugs). Where such effects have been detected, they are typically restricted to specific and infrequent outcomes, such as endometrial cancer in patients receiving unopposed oestrogen replacement therapy (6) or angio-oedema in patients receiving an angiotensin-converting enzyme (ACE) inhibitor (13). Hence, while observational studies involving small numbers of cases may well suffice for the detection of very large treatment effects, the reliable detection of more moderate effects (such as those that might plausibly be expected for calcium antagonists on CHD, cancer and bleeding risks) requires studies involving both large numbers of cases and random assignment of treatments so that both random errors and systematic errors are small in comparison with any effects of treatment.

\section{Effects of Calcium Antagonists on Coronary Heart Disease Risk}

Blood pressure levels are directly and continuously related to the risks of initial major CHD events (14) as well as to the long-term risks of death from CHD after non-fatal myocardial infarction (15). Similarly, blood pressure levels are directly and continuously related to the risks of initial (14) and recurrent stroke (16). Moreover, antihypertensive treatment with regimens based on diuretics or beta-blockers (but involving a wide variety of other agents) has been shown in randomised trials to reduce the risks of major CHD events (both non-fatal myocardial infarction and deaths from CHD) as well as strokes in hypertensive patients with or without known CHD or cerebrovascular disease $(7,8)$. Additionally, some agents that lower blood pressure have also been shown to reduce the risks of death and nonfatal myocardial infarction in patients with CHD, with or without hypertension. For example, there is strong evidence of this with beta-blockers (17), and there is emerging evidence that long-term treatment with ACE inhibitors may have similar effects (18). On the basis of the foregoing, it would seem reasonable to expect that other blood pressure lowering drugs should also reduce the risks of CHD events, unless such drugs had specific adverse cardiac effects that offset the benefits of blood pressure reduction. Some pathophysiological mechanisms have been proposed by which calcium antagonists may adversely affect the risks of CHD; these include pro-ischaemic, pro-arrhythmic, negative inotropic, hypotensive and reflex sympathetic effects $(19,20)$. However, the evidence is mixed as to whether calcium antagonists reliably produce such effects, and whether any such effects have important clinical consequences. The concerns that calcium antagonists may produce adverse cardiac effects are based primarily on outcome in patients treated with 
calcium antagonists in some randomised trials and observational studies.

\section{Randomised Controlled Trials \\ In patients with hypertension}

Data on the effects of calcium antagonists on major CHD events are available from several small randomised trials in patients with hypertension, none of which was designed specifically to assess the effects of treatment on such outcomes. Some of these trials were placebo-controlled studies of calcium antagonists $(21,22$, and Zhang $\mathrm{T}$, personal communication) and some compared the effects of calcium antagonists with other antihypertensive drugs $(21-24)$. Most of the studies investigated the effects of long-acting dihydropyridine drugs. But, although these trials collectively involved a total of about 6,000 patients, only about 50 cases of non-fatal myocardial infarction or death from CHD were observed during scheduled follow-up. Consequently, neither individually nor in combination did these trials document sufficient CHD events for the reliable determination of any plausible difference in risk between calcium antagonist and placebo groups or between calcium antagonist and other active treatment groups. So although CHD events were similarly distributed among calcium antagonist, other active treatment and placebo groups in these trials, the results do not preclude real differences in outcome-in either direction-of moderate (or even large) magnitude. Thus, the randomised trials of calcium antagonists in patients with hypertension provide little useful evidence from which to draw conclusions about the effects of these drugs on the risk of CHD.

One controlled trial of a long-acting formulation of nifedipine (which used alternate assignment to active treatment or placebo instead of proper random assignment) has recently been reported (25). However, in that study among 1,632 Chinese patients, only 4 cases of myocardial infarction were reported during 30 months of follow-up; strokes were less frequent among patients treated with nifedipine (16 vs. 36 strokes, relative risk 0.43 ; $95 \%$ CI $0.24-$ $0.77)$. A similar effect on stroke was also observed in one of the randomised controlled trials conducted in China (14 strokes among patients assigned nifedipine vs. 28 among controls; relative risk $0.49 ; 95 \%$ CI 0.26-0.92) (Zhang $\mathrm{T}$, personal communication), with very few strokes observed in the other randomised trials in hypertension.

\section{In patients with coronary heart disease}

A larger number of randomised trials of calcium antagonists has been conducted in patients with a history of CHD (mostly myocardial infarction). Some of these trials began treatment in the acute phase of myocardial infarction, while others started treatment later after infarction or among patients with stable angina. These trials fall into two main groups: trials of dihydropyridine drugs [mainly immediate release (IR) nifedipine] or verapamil or diltiazem, vs. untreated controls, and trials comparing the effects of calcium antagonists $v s$. beta-blockers.

In the first subgroup of studies, some (such as the DAVIT-II study) (26) demonstrated trends towards a reduction in CHD events in patients assigned a calcium antagonist and others (such as the TRENT study) (27) demonstrated trends towards an increase in events, but none demonstrated any clearly significant effect of calcium antagonists on the incidence of major CHD events. Overviews of these controlled trials of calcium antagonists have involved data from a total of about 20,000 patients, among whom data on myocardial infarctions (some of which led to death) were obtained from about $85 \%(28-30)$. In these overviews, data on total mortality were obtained from almost all patients, but CHD deaths were not analysed separately and no summary data are therefore available about the effects of treatment on the overall risk of myocardial infarction or death from CHD. In the studies contributing to these overviews, about 600 cases of myocardial infarction and 2,000 deaths were observed during the scheduled period of treatment and follow-up (but with some "double-counting" of these events because, in several studies, myocardial infarctions followed by death were not reported separately from myocardial infarctions with survival to the end of follow-up).

The results of these overviews provide no clear evidence of an effect of calcium antagonists, considered collectively, on the risks of myocardial infarction or on overall mortality. Post hoc exploration in subgroups of these trials did not find significant heterogeneity between the effects of the different individual agents. A non-significantly increased risk of myocardial infarction was observed in patients assigned a dihydropyridine (odds ratio $1.19 ; 95 \% \mathrm{CI}$ $0.92-1.53$ ) and a marginally significant decreased risk was observed in patients assigned verapamil or diltiazem (odds ratio 0.79; 95\% confidence limits $0.67-0.94)$. These trends for myocardial infarction were mirrored by similar trends in total mortality (but these analyses are not independent of each other, for in some trials, cases of myocardial infarction that led to death were included in both analyses). The greater apparent CHD risk in patients assigned a dihydropyridine mainly reflected the results of trials of nifedipine, while the lower risk in patients assigned a non-dihydropyridine agent reflected similar trends in trials of treatment with verapamil and with diltiazem. But although this indirect comparison of the effects on CHD in trials of dihydropyridine agents and those in trials of other calcium antagonists appeared to be conventionally significant (uncorrected $p$-value 0.01), this comparison may have been inflated by chance and selection due to the post-hoc nature of the observation. Notably, the similar comparison for mortality was not statistically significant. Since publication of these overviews, a further randomised trial of verapamil in patients with a history of myocardial infarction has been reported (31); this trial produced results that were consistent with a beneficial effect of ver- 
apamil on the risk of reinfarction, but they were not by themselves definitive. The addition of data from this study to the calcium antagonist trial overview strengthened slightly the evidence suggesting a beneficial effect of verapamil on the risks of major CHD events (relative risk 0.81, 95\% CI 0.67-0.98) (30), but the selective addition of this trial alone to the overview may have been influenced by its results and could therefore be biased.

Further emphasis on this dihydropyridine $v s$. nondihydropyridine comparison subsequently led to an analysis restricted solely to trials of nifedipine (19, 20 ). This involved data from about 10,000 patients in whom a total of about 750 deaths were observed. Analyses were restricted to all-cause mortality, and separate analyses of myocardial infarction or death from CHD were not provided. There was a slight excess of death (odds ratio 1.16, 95\% confidence limits 1.01-1.33) among patients assigned treatment with nifedipine. In the interpretation of this overview, emphasis was placed on the finding that most of the apparent increase in risk was in trials employing very high doses of nifedipine $(80 \mathrm{mg}$ or more per day). However, the number of events in this subset of the trials is small (only 96 deaths in total) and the hypothesis of an adverse effect of high-dose nifedipine represents the result of a series of selective post-hoc decisions to focus, first on dihydropyridine drugs alone, second on nifedipine specifically, and then finally on doses of $80 \mathrm{mg}$ or greater per day. [The hypothesis that higher doses might be associated with greater risk was based on observational studies in which the observed risk was increased among patients taking more than the median dose of nifedipine ( $40 \mathrm{mg}$ daily). But, if $\geq 40$ $\mathrm{mg}$ had been used to define high dose in the analysis of the randomised trials, then all of the trials would have been included in this category, and the findings would have been far less striking.] Thus, while the observation could be interpreted as an adverse dose-response relationship between nifedipine and the risk of death, the possibility that such a result could have been produced or exaggerated by data-dependent selection and the play of chance cannot be dismissed.

A separate overview of 24 short-term (average duration 10 weeks), placebo-controlled, randomised trials of a variety of different calcium antagonists in patients with stable angina was conducted using all of the data from safety and efficacy studies submitted to the US Food and Drug Administration for drug registration purposes (32). These studies involved a total of 1,450 patients among whom about 80 were withdrawn from treatment because of cardiovascular events (defined as increased or unstable angina, myocardial infarction, cardiovascular or unwitnessed death, ventricular tachycardia, other arrhythmias, or other cardiovascular dropout). The likelihood of treatment withdrawal for these reasons was somewhat greater in the patients assigned a calcium antagonist than in those assigned placebo (odds ratio $1.63 ; 95 \%$ CI 1.02-2.59). However, no data were given separately for myocardial infarction or CHD death, or for different types of calcium antagonists.

The other group of randomised trials in patients with CHD directly compared the effects of calcium antagonists $v s$. beta-blockers. Two recent trials of moderate size $(33,34)$ have compared the effects of these drugs in patients with stable angina. Neither of these studies showed any clear difference between agents in their effects on CHD risk, but once again these studies were too small (only about 100 CHD events observed among 1,500 patients) to detect plausible differences reliably between these treatments.

In summary, therefore, the results of all these trials of calcium antagonists in patients with CHD do not provide clear evidence of either a beneficial effect or an adverse effect of these agents on the risk of major CHD events in patients with established CHD. For although, in the placebo-controlled studies, there is a trend towards a beneficial effect of non-dihydropyridine agents on the risk of myocardial infarction and a trend towards an adverse effect of the dihydropyridine drugs studied (mostly IR nifedipine), neither can be said to provide definitive evidence of an effect of calcium antagonists on overall CHD risk. Nor are there any reliable data available from trials in patients with CHD about the effects of the slow-release or longacting dihydropyridine calcium antagonists on the risk of major CHD events.

\section{In patients with heart failure}

The other group of trials that have provided evidence about the effects of calcium antagonists on CHD risk are those conducted in patients with heart failure. There have been two studies of moderate size and duration $(35,36)$ and several smaller studies. The two larger studies are placebo-controlled trials of newer dihydropyridine agents (felodipine in one and amlodipine in the other). Complete data on non-fatal myocardial infarction and fatal CHD events were not available from either trial; one study provided data on CHD deaths. but not on non-fatal myocardial infarction and the other provided data on non-fatal myocardial infarction but not on CHD deaths. The $36 \mathrm{CHD}$ events that were reported in total in these two studies were, however, approximately evenly distributed between treatment and control groups. Clearly, however, the unavailability of complete data on major CHD events together with the small number of events actually reported limit the ability of these trials to detect plausibly modest increases or decreases in CHD risk with calcium antagonist treatment. One of these studies did, however, observe an increase in the risk of pulmonary oedema among patients assigned treatment with amlodipine (36); while there was no evidence of such an increase in risk in the trial of felodipine $(35,36)$, a smaller study of this drug reported an increase in treatment withdrawal due to worsening heart failure (37). 


\section{Observational Studies}

\section{Case-control studies}

Three case-control studies have reported data about the risks of myocardial infarction or death from CHD among hypertensive patients treated with a calcium antagonist. One of these studies observed an increased CHD risk in patients treated with a calcium antagonist (38) and two did not $(39,40)$. Among those not reporting an increase in risk, one included about 200 cases and 800 controls (39) and the other included about 100 cases and 300 controls (40). Both of these studies were too small to detect even a doubling in the risk of CHD with calcium antagonists. The case-control study that reported an increase in risk involved about 600 cases and 2,000 controls (38). Among patients receiving treatment with a calcium antagonist, the risk of a major CHD event was about $60 \%$ greater than that observed in patients treated with other drugs. The increased risk primarily reflected a higher risk of CHD events among those patients treated with a non-dihydropyridine calcium antagonist. This study also showed that patients receiving treatment with a calcium antagonist were more likely than others to have a previous history of CHD or other risk factors, such as diabetes. The higher risk of CHD events among patients treated with calcium antagonists persisted after attempts to adjust for disease history and risk factor profile. A further case-control study has recently reported data about the risks of a variety of cardiovascular events (but not CHD risks separately) among hypertensive patients treated with a calcium antagonist (41). That study included about 200 cases and a similar number of controls, and reported no overall increase in risk among individuals receiving treatment with a calcium antagonist (odds ratio $0.89,95 \%$ CI $0.45-1.75$ ). However, while there was no evidence of increased risk among patients treated with a long-acting calcium antagonist an increased risk was observed among patients taking a short-acting calcium antagonist (odds ratio 3.98, 95\% CI 1.18-13.89). However, the prevalence of prior cardiovascular disease was much higher among patients receiving treatment with a short-acting calcium antagonist $(63 \%)$ than among those receiving treatment with a long-acting calcium antagonist $(38 \%)$ or another non-calcium antagonist antihypertensive drug $(21 \%)$. While an increased risk among patients treated with a short-acting calcium antagonist was still evident among those patients without such a disease history, the extremely large size of the relative risk (12.4) and the small number of individuals treated with a short-acting calcium antagonist in this subgroup (9 cases and 1 control) raises concerns about the reliability of the result.

As discussed above in the section on Methodological Principles, the major concern about such observational studies of treatment outcome is the large potential for systematic error to affect the results. It is possible, indeed likely, that the decision to prescribe a calcium antagonist specifically for the treatment of high blood pressure was frequently determined, at least in part, by factors associated with
CHD risk (such as disease history, age, risk factors levels). Thus, the use of hypertensive patients as controls will not necessarily avoid confounding by indication. While the statistical analyses of these studies included adjustment for some potential confounding factors, this provides little reassurance that "indication bias" is not a plausible alternative explanation for the observed findings. For, such statistical adjustment is likely to be incomplete due both to the error with which confounding factors (such as disease history or risk factor levels) were measured and to the limited number of potential confounding factors for which data were available (e.g. typically, little data were available about evidence of subclinical atherosclerotic or myocardial disease that may affect prescribing patterns). Even comparisons of risk in patients treated with a calcium antagonist and patients treated with a beta-blocker may not be free of indication bias. Because, although both treatments may be selectively given to high-risk patients (perhaps for the treatment of angina), there is no particular reason to expect that the average CHD risk (in the absence of treatment) in patients treated with a calcium antagonist should necessarily be the same as that in patients treated with a betablocker. Moreover, the suggestion of a dose-response association, while consistent with the hypothesis of a true adverse effect of calcium antagonists on CHD risk, could also be explained by the preferential use of higher doses of calcium antagonists in very high risk patients, perhaps for the control of higher blood pressures (42) or more refractory angina.

\section{Cohort studies}

An increased risk of CHD events among patients treated with a calcium antagonist has also been reported in an analysis of a small subgroup of about 900 participants from a prospective study of more than 10,000 elderly individuals (43). The subgroup for which data were reported included only patients treated with a single antihypertensive agent and excluded patients treated with multiple agents and other participants not receiving antihypertensive therapy. In this subgroup, there was a total of about $50 \mathrm{CHD}$ deaths observed during follow-up and, among those without a history of CHD, there were only about 60 fatal and non-fatal CHD events in total. The distribution of these events suggested a several-fold greater risk of CHD events among those treated with nifedipine or with diltiazem (but not verapamil). As in some of the case-control studies described above, patients treated with a calcium antagonist in this study population were likely to have a higher-risk medical history and a worse risk factor profile than patients treated with other drugs. Although the increased risk persisted after controlling for some potential confounding factors, it is, once again, likely that the observed higher risk of CHD events in patients treated with a calcium antagonist reflects, at least in part, the selection of higher-risk patients for such treatment. The size of the risk observed among patients treated with calcium antagonists in this study population is implau- 
sibly large, and inconsistent with the available results from randomised trials of calcium antagonists in patients with CHD. This underlines the magnitude of the biases that can affect such non-randomised studies.

Three other cohort studies have recently reported on the risk of all-cause mortality (but not specifically the risks of CHD events) among patients with a history of CHD receiving treatment with calcium antagonists. In one study of 1,200 patients with myocardial infarction (44), overall mortality rates appeared to be higher among patients treated with either nifedipine or verapamil. In another study of about 11,000 patients with established CHD, half of whom were treated with calcium antagonists (half with nifedipine and one third with diltiazem), there was no clear increase in risk associated with calcium antagonist treatment (45). Once again, however, in both these studies there was evidence that patients treated with calcium antagonists tended to have a worse medical history and/or risk factor profile than did others, and the possibility that either of the results could have been importantly affected by confounding is, again, not easily dismissed. Most recently, a report on 7 years of follow-up in the Framingham Study provided no evidence of increased mortality among 400 hypertensive patients (half with a history of CHD) treated with a calcium antagonist (46).

\section{Effects of Calcium Antagonists on Cancer Risk}

In some cohort studies, a modest direct association between blood pressure levels and the overall risk of death from cancer has been reported (47). However, analyses based on the totality of evidence from a large number of cohort studies have not demonstrated such an association (Prospective Studies Collaboration, unpublished data). While complete data on cancer incidence are not available from the earlier trials of antihypertensive treatment, those studies have demonstrated no change in the risk of death from non-vascular causes among patients assigned active treatment, mostly with diuretic- or beta-blocker-based regimens $(7,8)$. Similarly, no complete or reliable data on cancer incidence are available either from the more recent randomised trials of calcium antagonists in patients with hypertension or from the trials of calcium antagonists in patients with CHD. A review by the US Food and Drug Administration (Albert DeFelice, personal communication) of all the available evidence concerning rodent tumorigenicity concluded that there was no evidence of any direct carcinogenic effect of any registered calcium antagonist in any of several strains of both rats and mice, and no indication of genotoxicity in a variety of bacterial and mammalian cells calibrated with known mutagens. While there has been a report that verapamil increased the in-vitro growth of human colon and breast cancer cell lines (48), the main concerns about possible adverse effects of calcium antagonists on cancer risk have arisen primarily from the results of some observational studies of patients treated with a calcium antagonist.

\section{Observational Studies}

Two reports $(49,50)$ from part of the same parent cohort as the smaller study on calcium antagonist use and total mortality referred to above (43) and so involving some of the same people describe a somewhat greater incidence of cancer among patients receiving treatment with a calcium antagonist than among others in the population studied. This cohort study involved a total of 5,052 elderly individuals followed for 4 years. The first report was based on a total of 61 cases of cancer, 27 of which occurred among patients treated with calcium antagonists. The second report was based on a total of 420 cases, 47 of which occurred among patients treated with a calcium antagonist. Thus neither report from this study involved sufficient cancer cases to characterise reliably any plausibly modest association between calcium antagonist use and overall cancer risk. Moreover, the two reports do not provide independent evidence about the association, since it appears that the cancer cases included in the first analysis were also included in the second, and in the report based on the larger number of cases, the observed increase in the crude rate of cancer among calcium antagonist-treated patients was of only borderline statistical significance ( $p$-value $0.03)$. Multivariate-adjusted analyses of the data suggested a greater increase in cancer risk with calcium antagonist use (adjusted odds ratio 1.72; 95\% CI 1.27-2.34). This increase in risk was observed in patients treated with either nifedipine or verapamil, but did not appear to reflect an increase in any one type of cancer (or subgroup of related cancers). As in some of the observational studies of CHD, there also appeared to be a direct association between the dose of calcium antagonist used and risk. It was suggested that these findings could be explained by a cancer-promoting effect of calcium antagonists, mediated perhaps by inhibition of apoptosis. While there is evidence that calcium antagonists can inhibit experimentally induced apoptosis $(51,52)$, and increase the in-vitro growth of certain human cancer cell lines (48), other data on the effects of calcium antagonists on apoptosis and growth in a tissue with increased proliferation indicate stimulation of apoptosis and inhibition of proliferation (53).

As in the observational studies of calcium antagonists and CHD, confounding by indication is also a plausible alternative explanation for the findings in this study of calcium antagonists and cancer. At entry to the study, there was evidence of a more frequent history of ill health among patients taking a calcium antagonist. Although the increased risk of cancer persisted after controlling for some aspects of medical history and a few other relevant factors that had been recorded (such as smoking, alcohol consumption and body mass index), for reasons given above and elsewhere (54), such analyses can not fully exclude confounding by indication as an alternative explanation. Residual confounding would be consistent with the observation that the 
association was not with just one or a few types of cancer, but of a generalised increase in most cancer types. Established exogenous causes of cancer typically affect particular sites selectively, and the apparently broad association of calcium antagonist use with cancer risk in this study population could be explained by more frequent use of these agents among sicker patients generally, including some whose symptoms were the consequence of undiagnosed cancer. Moreover, the apparent absence of the usual latent period between exposure and cancer development is also atypical of established exogenous causes of cancer, and would once again suggest that residual confounding may be an explanation.

It has also been observed that sicker patients - as were the patients treated with a calcium antagonist in this study - are typically less likely to relocate than others who are in better health (54). For this reason it is possible that there may be a bias towards the less frequent detection not only of cancers but also of other conditions (such as myocardial infarction) among healthier individuals. Additionally, sicker patients could be more likely to receive diagnostic investigations that uncover malignancies, and this could also introduce detection bias, particularly over the short duration of these studies. Both these sources of detection bias could result in a spurious apparent increase in risk among sicker patients, such as those receiving treatment with a calcium antagonist.

One case-control study of colon cancer has recently reported an extreme increase in risk among patients treated with verapamil but not with other calcium antagonists. However, only 11 patients in the entire study population reported taking this drug (55). In the cohort study described above there was no significant increase in colon cancer among patients receiving treatment with any calcium antagonist, and separate data were not provided about the risk in patients receiving verapamil.

\section{Effects of Calcium Antagonists on Bleeding Risk}

It has been suggested that calcium antagonists might cause bleeding by inhibiting platelet aggregation while also preventing the normal vasoconstrictive response to bleeding (56). A randomised placebocontrolled trial of the dihydropyridine drug, nimodipine, was stopped prematurely after 149 of a scheduled 400 patients undergoing cardiac valve replacement had been recruited, due to a few more deaths having been observed among those allocated nimodipine $[8 / 75(11 \%)$ vs. $1 / 74(1 \%)](57)$. As major bleeding during and after surgery appeared to occur often in that study, analyses of bleeding were performed which indicated an excess of major bleeding with nimodipine [10 (13\%) vs. $2(3 \%) ; p$ value 0.03$]$. Subsequently, four observational studies explored the associations between calcium antagonist use and bleeding $(56,58-60)$.

\section{Observational Studies}

The first of these was a cohort study that included a total of 120 individuals with gastrointestinal (GI) bleeds, of which 47 were considered to be severe, among a total population of 1,636 individuals aged 68 or older taking beta-blockers, calcium antagonists or ACE inhibitors for any indication (56). Compared with beta-blocker use, the use of any calcium antagonist was associated with an increased risk of any GI bleeding (42 bleeds: relative risk 1.86; 95\% CI 1.22-2.82) and of severe GI bleeding (31 bleeds: relative risk 1.68; 95\% CI 1.03-2.74). Surprisingly, this was similar to the excess risk observed with aspirin use (relative risk 1.5) and with oral anticoagulants (relative risk 2.2). This apparent increase in any GI bleeding was observed with the use of diltiazem (18 bleeds: relative risk 2.18; 95\% CI 1.24-3.82) and verapamil (13 bleeds: relative risk 2.39 ; $95 \%$ CI 1.28-4.44) but not with nifedipine (9 bleeds: relative risk $1.04 ; 95 \%$ CI 0.51-2.13).

A similar, though non-significant, increase in the risk of GI bleeding was also observed with calcium antagonist use (odds ratio $1.87 ; 95 \%$ CI $0.79-4.41$ ) in a study of 73 cases and 73 age- and sex-matched controls; however, in that study $70-80 \%$ of patients treated with calcium antagonists were using nifedipine (58). By contrast with these studies, a large comparison of outcome between 2,248 calcium antagonist-treated patients undergoing cardiac surgery and 2,909 cardiac surgery patients not receiving calcium antagonists did not indicate any increases in transfusions (about 200 in total: 3\% on calcium antagonist vs. 5\% not) or reoperations for bleeding (about 100: 1\% vs. 2\%); indeed, there were significant trends towards fewer such bleeds with calcium antagonist use (59). Similarly, in a small series of 120 patients undergoing coronary artery graft surgery, calcium antagonist use was not associated with any increases in post-operative bleeding or transfusions (60).

\section{Randomised Trials}

In patients with coronary heart disease

Most randomised trials of calcium antagonists in patients with CHD have not specifically provided data on bleeding. But, review of the published reports of several of the larger randomised controlled trials of calcium antagonist use - involving a total of over 15,000 patients with an average follow-up of about 11 months (i.e. about 14,000 patient-years of treatment) $(26,27,31,36,61-63)$ - indicates that no bleeding excess was reported in any of these controlled trials, although detailed reasons for discontinuing study treatment were given. This lack of any reported bleeding risk in these large randomised trials does not seem to be consistent with the excess risk of GI bleeding of about 1 case per 100 patientyears of calcium antagonist treatment estimated from the first of the observational studies described $(56,64)$. 
In patients with cerebrovascular disease

Calcium antagonists have been assessed extensively in randomised controlled trials among patients in whom any adverse effects on bleeding would have been expected to be particularly obvious - for example, in the immediate management of subarachnoid haemorrhage and acute ischaemic stroke. In the management of subarachnoid haemorrhage, the calcium antagonists that have been most widely studied in randomised trials are the dihydropyridines (in particular nimodipine), and these have usually been started as soon as possible after the initial bleed. A meta-analysis of 6 randomised placebo-controlled trials of 3-12 weeks of nimodipine in over 1,200 such patients indicated a reduction in the incidence of "poor outcome" (i.e. dead/ vegetative/severely disabled) (65), and in the largest of these studies, among over 500 patients, it was explicitly reported that there was no excess of any adverse effect such as increased rebleeding (66). Studies of other calcium antagonists in acute subarachnoid haemorrhage have been much smaller, although larger studies are in progress that might help further to resolve any remaining concerns about bleeding with calcium antagonist use.

In acute ischaemic stroke, both anticoagulant and fibrinolytic therapies have been shown to be associated with haemorrhagic transformation and intracerebral bleeds, leading to a poor outcome, so trials of calcium antagonists in this setting might be expected to be especially useful for demonstrating (or refuting) the sort of hazards due to bleeding that have been suggested by the results of some observational studies. A meta-analysis has been reported involving nine randomised placebo-controlled trials in which a total of about 3,700 stroke patients were allocated $2-4$ weeks of $120 \mathrm{mg}$ daily oral nimodipine or control (67). Overall in this overview there was no evidence of any adverse effects of calcium antagonist use on death or clinical deterioration; indeed, subgroup analyses suggested improved outcome with nimodipine among those treated within $12 \mathrm{~h}$ of symptom onset, albeit with an opposite trend among those treated after $24 \mathrm{~h}$. But, in a more recent trial of nimodipine $120 \mathrm{mg}$ daily in 350 acute ischaemic stroke patients there did not appear to be any improvement in functional outcome, even among those treated within $12 \mathrm{~h}$, and mortality was significantly increased at 1 and 3 months (68). Data on bleeding were not explicitly reviewed in the meta-analysis and there were no bleeds reported in the more recent trial, but more detailed consideration of such information in each of these trials (along with that in trials of other calcium antagonist regimens) might provide an opportunity to assess any haemorrhagic complications of calcium antagonists in this setting of high bleeding risk. Selective emphasis on apparent increases (or decreases) in bleeding in just one or other of these stroke studies may, however, be biased as the studies that report such information may have extreme results.

\section{Summary and Conclusions}

Calcium antagonists are used extensively throughout the world for the treatment of high blood pressure and angina. In Japan, they are prescribed for about three-quarters of all treated hypertensive patients (69) and in China, calcium antagonists (mostly short-acting agents) are prescribed for about onehalf of all such patients (Liu L, personal communication). In these Far East countries, the main objective of antihypertensive treatment is the prevention of stroke, since the risks of CHD are very much lower. For this reason, the concerns that have been raised about the effects of calcium antagonists - particularly the short-acting agents - on CHD risk are less relevant to their use in the Far East than in the West. In Europe and the USA, calcium antagonists are prescribed for a smaller but still substantial proportion of patients with high blood pressure. The major objectives of antihypertensive treatment in these populations are the prevention of both stroke and CHD events. However, at the present time, data on the effects of calcium antagonists on stroke and CHD are available from only four small randomised trials in patients with hypertension, and even in aggregate the data from these trials do not provide reliable information about effects on these important outcomes of treatment. There are more data on CHD events from trials in patients with myocardial infarction or angina, but overviews of the results from these trials do not show a clear overall effect of calcium antagonists on the risks of myocardial infarction or death from any cause. Selective emphases on subgroups of trials with particular agents suggest the possibility of modestly beneficial effects of diltiazem and verapamil and modestly adverse effects of nifedipine. But these trends were not strong, and could well be biasedboth in favour of and against particular agents - as a consequence of the retrospective manner in which they were identified. The limited information available from trials of calcium antagonists in patients with heart failure adds little to the assessment of the effects of these agents on the risk of CHD.

Although the data on CHD risk from randomised trials of calcium antagonists are limited, they do exclude the possibility of adverse effects of the extreme magnitude suggested in reports from some observational studies. For example, the upper confidence limit of the estimated effect of nifedipine in randomised trials in patients with CHD includes an adverse effect of $30-50 \%$. This is substantially less than the $60-300 \%$ increases in risk suggested by some observational studies of hypertensive patients, underlining the very large potential for bias in such studies, and suggesting that their results may be largely, if not wholly, due to confounding rather than to drug effects. There is clear evidence from several studies that calcium antagonists are preferentially prescribed to the sickest patients, including those with CHD and other high-risk conditions such as diabetes, and it is unlikely that statistical 
adjustment for confounding can remove this source of bias entirely.

The available evidence does not prove the existence of either beneficial or harmful effects of calcium antagonists on the risks of major CHD events, including fatal or non-fatal myocardial infarctions and other deaths from CHD. This applies to the evidence on all calcium antagonists considered collectively, and to that on subgroups of these agents.

Evidence about the effects of calcium antagonists on the risk of cancer is derived almost entirely from observational studies, since no reliable data have been reported on this outcome from randomised trials. In this situation, it is particularly difficult to test the validity of reports from some observational studies suggesting an increased cancer risk among patients treated with a calcium antagonist. The observed increases in cancer risk could reflect a chance occurrence, systematic error or a true adverse effect of these agents. The very small numbers of cancer cases on which the findings of increased risk were based and the modest level of statistical significance are consistent with chance being an explanation. The results may well also have been affected by confounding by indication, since patients in poor health appear to be given calcium antagonists more frequently than other antihypertensive drugs. Detection bias may also have occurred, with adverse outcomes more likely to be detected among those prescribed calcium antagonists, who as a consequence of their poorer state of health may be both less likely to relocate and more likely to receive diagnostic procedures. The possibility of residual confounding, even after statistical adjustment, is consistent with the observation that the increased risk appears to be distributed widely across cancer subtypes, as well as between other distinctly different disease entities [including gastrointestinal haemorrhage (56) and Parkinson's disease (70)]. While a biological mechanism has been suggested to account for the increased cancer risk in patients treated with a calcium antagonist, this is highly speculative and controversial (71), and adds little to the strength of the evidence relating calcium antagonist use to cancer incidence. In particular, it is inconsistent with the evidence from animal studies demonstrating no carcinogenicity of any calcium antagonist approved for use in the USA. In considering the evidence about cancer risk, there is cause to reflect on earlier reports from observational studies raising concerns about a possible adverse effect of reserpine (72-74), another blood pressure lowering drug, on breast cancer risk. The observation was subsequently shown to be the consequence of confounding (75). Other reports of an increased cancer risk associated with atenolol (76) and enalapril (77) remain unconfirmed, but they too seem likely to have been the consequence of chance and selective post-hoc emphasis.

The available evidence from observational studies does not provide good evidence of an adverse effect

\section{of calcium antagonists on cancer risk.}

The available evidence on bleeding risk associated with calcium antagonists does not indicate a consistent pattern of hazard. Instead, excesses of bleeding associated with calcium antagonist use observed in some small observational studies and randomised trials have not been observed in some other, larger, studies that have addressed this issue. Moreover, calcium antagonists have been carefully studied in certain circumstances where the risks associated with bleeding are high, and yet no excesses of severe bleeds or adverse outcome due to bleeding were reported. So, although it is not possible to rule out modest effects of calcium antagonists on bleeding, the available evidence does not seem to be consistent with the large haemorrhagic risk (i.e. similar to that associated with oral anticoagulants) that has been suggested by some investigators.

The available evidence from observational studies and randomised trials does not provide clear evidence of an adverse effect of calcium antagonists on bleeding risks.

Two major issues are raised by the findings of this review. The first is the clear failure of pharmaceutical companies, regulatory authorities and clinical researchers to ensure the timely conduct of studies that could provide clinicians and patients with reliable evidence about both the safety and efficacy of widely prescribed calcium antagonists. Such assurance requires studies in which both random and systematic errors are minimised concurrently, and this can only be provided by large-scale randomised trials. A number of such trials are now underway, some of which were ongoing at the time concerns about the safety of calcium antagonists were raised, and some of which were initiated in direct response to the controversy. By early in the next decade, data should be available from trials involving about 100,000 patients with hypertension randomised to treatment with a calcium antagonistbased regimen or a diuretic/beta-blocker-based regimen. About half of these patients will be in trials of the newer slow-release or long-acting dihydropyridine agents, and the remainder will be in trials of non-dihydropyridine drugs. These studies should be able to determine reliably whether such calcium antagonist-based regimens have any importantly different effects on CHD and major bleeding risks from those conferred by diuretic- or beta-blockerbased regimens. Long-term follow-up of patients in these trials beyond the scheduled treatment period could also provide useful information about the effects of these agents, if any, on cancer risk. Such large-scale studies of efficacy and safety should routinely be initiated early in the development of new drug classes that are destined to be marketed for the treatment of common cardiovascular diseases. Only in this way will controversies of the kind that has involved calcium antagonists be avoided in the future. 
The second issue raised by the findings of this review is the major role played by observational studies in generating public concerns about possible adverse effects of calcium antagonists. It has recently been suggested that, in the absence of randomised trials, observational studies can provide useful information about treatment effects $(78,79)$. However, the results of previous observational studies of medical interventions indicate that unless the observed effect of treatment is very large, the results of such studies do not provide a reliable guide to the size, or even the direction, of the real treatment effects. This is probably, in large part, due to confounding by indication, but it may also reflect detection bias and publication bias. It is quite possible that there are a number of other observational studies with data on both calcium antagonist use and cardiovascular and cancer outcomes that remain unpublished. Since negative findings are less likely to be published than positive findings $(80)$, it is questionable whether the currently available data are representative of the totality of the observational data potentially available.

In conclusion, the available evidence about the effects of calcium antagonists on the risks of CHD, cancer and bleeding does not establish the existence of beneficial or harmful effects. This conclusion has few implications for current guidelines about the treatment of hypertension, since most already acknowledge the absence of reliable randomised evidence about the effects of calcium antagonists on major morbidity and mortality. For the treatment of angina, the results of this review similarly do not provide any strong reason to change recommendations. However, they do underline the large differences that exist in the strength of the evidence available about the effects of different classes of blood pressure lowering drugs on major health outcomes. For patients with hypertension, there is now very strong evidence from randomised trials that treatment regimens involving diuretics or betablockers (in combination with a wide variety of other older antihypertensive agents) are effective in reducing the risks of major CHD events and stroke, with no effect detected on major non-vascular causes of death (of which there were, in aggregate, several hundred) during the 5-year average duration of these trials $(7,8)$. For patients with a history of myocardial infarction, there is strong evidence from randomised trials that treatment with a beta-blocker reduces the risks of death and re-infarction (17), and in the presence of left ventricular dysfunction or heart failure, there is clear evidence from randomised trials of beneficial effects of ACE inhibitors on overall mortality and heart failure-related morbidity (81), and emerging evidence of benefit for major CHD events (18). However, for other patient groups (including those with hypertension), there is still no direct evidence of benefit from ACE inhibitors for major CHD events or for stroke. By contrast, there is little or no reliable evidence from randomised trials of beneficial (or harmful) effects of calcium antagonists on major cardiovascular mor- bidity and mortality in any patient group. For, although the trends for verapamil and diltiazem in patients with a history of myocardial infarction are suggestive of benefit and the trends for nifedipine are suggestive of harm, selective emphasis on these subgroups of agents was largely retrospective and the trends could therefore be inflated (or produced entirely) by chance and selection. Hence they require confirmation in further trials. For other newer agents, such as alpha-blockers and angiotensin-II antagonists, there is no evidence whatsoever about their effects on major cardiovascular events in any patient group. Whether calcium antagonists or the other newer agents have similar, lesser or greater effects on major cardiovascular outcomes than do the older established treatments will not be known for several more years, when the ongoing trials are completed.

\section{Acknowledgements}

\section{Ad Hoc Subcommittee Membership}

Members of this subcommittee were appointed by the Liaison Committee of the World Health Organisation and the International Society of Hypertension. They were chosen to represent a range of experience, viewpoints and geographic regions. In order for readers of this review to take account of any potential conflicts of interest among members of the subcommittee, each member was asked to declare relevant financial interests including research supported by pharmaceutical companies*, current consultancies for such companies ${ }^{\dagger}$, and shares in any such companies. Details of all such interests have been provided to the Liaison Committee of the World Health Organisation and International Society of Hypertension. No committee member held shares in any pharmaceutical company, a few held consultancies, and most received support for research from a variety of companies.

Subcommittee members: Michael Alderman* (New York), Kikuo Arakawa (Fukuoka), Lawrie Beilin*, $\dagger$ (Perth), John Chalmers* (Sydney), Jay Cohn* (Minneapolis), Rory Collins* (Oxford), Robert R Fenichel(Washington), Edward D Frohlich* (New Orleans), Pavel Hamet*, (Quebec), Lennart Hansson*, $\uparrow$ (Uppsala), Charles Hennekens* (Boston), Emilio Kuschnir (Cordoba), Liu Lisheng* (Beijing), Stephen MacMahon* (Auckland), Giuseppe Mancia*,† (Milan), Ingrid Martin (Geneva), Joel Menard* (Paris), Peter Sleight* (Oxford), Judith Whitworth (Sydney), Salim Yusuf* (Hamilton), Alberto Zanchetti* ${ }^{*}$ (Milan).

Writing committee: Stephen MacMahon, John Chalmers, Rory Collins, with the assistance of Bruce Neal (Auckland).

\section{Sponsorship}

The activities of this subcommittee were underwritten by the International Society of Hypertension and were sponsored by several non-commercial organisations including the British Heart Foundation, the Canadian Hypertension Society, the Foundation for High Blood Pressure Research (Australia), the High Blood Pressure Research Council of Australia, the Medical Research Council of 
Canada, the Metabolic Disease Foundation of Japan, the National Health Committee of New Zealand, the National Health and Medical Research Council of Australia, the National Heart Foundation of Australia and the Swedish Society of Hypertension. No pharmaceutical company provided support for the activities of this subcommittee.

\section{References}

1. Collins R, Peto R, Gray R, Parish S: Large-scale randomized evidence: trials and overviews, in Weatherall D, Ledingham J, Warrell D(eds): Oxford Textbook of Medicine. Third Edition. Oxford, Oxford University Press, 1996, 21-32.

2. Collins R, Gray R, Godwin J, Peto R: Avoidance of large biases and large random errors in the assessment of moderate treatment effects: the need for systematic overviews. Stat Med 1987; 6: 245-250.

3. Bigger J, Fleiss J, Rolnitzky L, Merab J, Ferrick K: Effect of digitalis treatment on survival after acute myocardial infarction. Am J Cardiol 1985; 55: 623630.

4. Yusuf S, Garg R, Smith T, et al: Which heart failure patients benefit the most from long-term digoxin therapy. Circulation 1996; 94: I-23.

5. Yusuf S, Furberg C, Wittes J, Bailey K: Digitalis-a new controversy regarding an old drug: the pitfalls of inappropriate methods. Circulation 1986; 73: 14-18.

6. Grady D, Rubin SM, Petitti DB, et al: Hormone replacement therapy to prevent disease and prolong life in postmenopausal women. Ann Intern Med 1992; 117: 1016-1037.

7. Collins R, Peto R, MacMahon S, et al: Blood pressure, stroke, and coronary heart disease. Part 2, short-term reductions in blood pressure: overview of randomised drug trials in their epidemiological context. Lancet $1990 ; 335$ : 827-839.

8. Collins R, MacMahon S: Blood pressure, antihypertensive drug treatment and the risks of stroke and of coronary heart disease. Br Med Bull 1994; 50: 272-298.

9. Antiplatelet Trialists Collaboration: Secondary prevention of vascular disease by prolonged antiplatelet treatment. Br Med J 1988; 296: 320-331.

10. Antiplatelet Trialists Collaboration: Collaborative overview of randomised trials of antiplatelet therapy. Part I: Prevention of death, myocardial infarction, and stroke by prolonged antiplatelet therapy in various categories of patients. $\mathrm{Br}$ Med $J$ 1994; 308: 7172.

11. Thumer $\mathbf{H}$, Lund-Larsen $\mathrm{P}$, Tverdal A: Is blood pressure treatment as effective in a population setting as in controlled trials? Results from a prospective study. J Hypertens 1994; 2: 481-490.

12. Paganini-Hill A, Chao A, Ross R, Henderson B: Aspirin use and chronic diseases: a cohort study of the elderly. $\mathrm{Br}$ Med $J$ 1990; 300: 116-118.

13. Israili $\mathrm{Z}$, Hall $\mathrm{W}$ : Cough and angioneurotic edema associated with angiotensin-converting enzyme inhibitor therapy. A review of the literature and pathophysiology. Ann Intern Med 1992; 117: 234-242.

14. MacMahon S, Peto R, Cutler J, et al: Blood pressure, stroke, and coronary heart disease. Part 1, prolonged differences in blood pressure: prospective observational studies corrected for the regression dilution bias. Lancet 1990; 335: 765-774.

15. Flack J, Neaton J, Grimm R, et al, for the Multiple Risk Factor Intervention Trial Research Group: Blood pressure and mortality among men with prior myocardial infarction. Circulation 1995; 92: 2437-
2445.

16. Rodgers A, MacMahon S, Gamble G, Slattery J, Sandercock P, Warlow C, on behalf of the UKTIA Collaborative Group: Blood pressure and risk of stroke in patients with cerebrovascular disease. $\mathrm{Br}$ Med J 1996; 313: 147.

17. Yusuf S, Peto R, Lewis J, Collins R, Sleight P: Betablockade during and after myocardial infarction: an overview of the randomized trials. Prog Cardiovasc Dis 1985; 27: 335-371.

18. Lonn E, Yusuf S, Prabhat J, et al: Emerging role of angiotensin-converting enzyme inhibitors in cardiac and vascular protection. Circulation 1994; 90: 20562069.

19. Furberg C, Psaty B, Meyer J: Nifedipine. Dose-related increase in mortality in patients with coronary heart disease. Circulation 1995; 92: 1326-1331.

20. Furberg C, Psaty B: Corrections to the nifedipine meta-analysis. Circulation 1996; 93: 1475-1476.

21. Neaton JD, Grimm RH, Prineas RJ, et al: Treatment of Mild Hypertension Study. Final results. J Am Coll Cardiol 1993; 270: 713-724.

22. Materson B: Department of Veteran Affairs single drug therapy of hypertension: final results. $A m J$ Hypertens 1993; 8: 189-192.

23. The GLANT Study Group: A 12-month comparison of ACE-inhibitor and Ca-antagonist therapy in mild to moderate essential hypertension - The GLANT Study. Hypertens Res 1995; 18: 235-244.

24. Borhani N, Mercuri M, Borhani P, et al: Final outcome results of the Multicenter Isradipine Diuretic Atherosclerosis Study (MIDAS). A randomized controlled trial. JAMA 1996; 276: 785-791.

25. Gong L, Zhang W, Zhu Y, Zhu J, 11 collaborating centres in the Shanghai area, Kong D, et al: Shanghai trial of nifedipine in the elderly (STONE). $J$ Hypertens 1996; 14: 1237-1245.

26. The Danish Study Group on Verapamil in Myocardial Infarction: Effect of verapamil on mortality and major events after acute myocardial infarction (The Danish Verapamil Infarction Trial-II-DAVIT II). $A m$ J Cardiol 1990; 66: 779-785.

27. Wilcox R, Hampton J, Banks D, et al: Trial of early nifedipine in acute myocardial infarction: the TRENT study. Br Med J 1986; 293: 1204-1208.

28. Held PH, Yusuf S, Furberg CD: Calcium channel blockers in acute myocardial infarction and unstable angina: an overview. $\mathrm{Br} \mathrm{Med} J$ 1989; 299: 1187-1192.

29. Yusuf S, Held P, Furberg C: Update of effects of calcium antagonists in myocardial infarction or angina in light of the Second Danish Verapamil Trial (DAVITII) and other recent studies. Am J Cardiol 1991; 67: 1295-1297.

30. Yusuf S: Verapamil following uncomplicated myocardial infarction: promising, but not proven. Am J Cardiol 1996; 77: 421-422.

31. Rengo F, Carbonin P, Pahor M, et al, and the CRIS Investigators: A controlled trial of verapamil in patients after acute myocardial infarction. Results of the Calcium Antagonist Reinfarction Italian StudyCRIS. Am J Cardiol 1995; 77: 365-369.

32. Glasser S, Clark P, Lipicky R, Hubbard J, Yusuf S: Exposing patients with chronic, stable, exertional angina to placebo periods in drug trials. JAMA 1991; 265: $1550-1554$

33. Dargie H, Ford I, Fox $\mathrm{K}$, on behalf of the TIBET study group: Total Ischaemic Burden European Trial (TIBET). Effects of ischaemia and treatment with atenolol, nifedipine SR and their combination on outcome in patients with chronic stable angina. Eur Heart J 1996; 17: 104-112. 
34. Rehnqvist N, Hjemdahl P, Billing E, et al: Effects of metoprolol vs. verapamil in patients with stable angina pectoris. Eur Heart J 1996; 17: 76-81.

35. Cohn J, Ziesche S, Loss L, Anderson G, and the VHeFT Study Group: Effect of felodipine on shortterm exercise and neurohormone and long-term mortality in heart failure: results of V-HeFT VIII. Circulation 1995; 92: I-143.

36. Packer M, O'Connor $\mathrm{C}$, Ghali $\mathrm{J}$, et al, for the Prospective Randomized Amlodipine Survival Evaluation Study Group: Effect of amlodipine on morbidity and mortality in severe chronic heart failure. $N$ Engl J Med 1996; 335: 1107-1114.

37. Littler W, Sheridan D, on behalf of the UK Study Group: Placebo controlled trial of felodipine in patients with mild to moderate heart failure. Br Heart $J$ 1995; 73: 428-433.

38. Psaty B, Heckbert S, Koepsell $\mathrm{T}$, et al: The risk of myocardial infarction associated with antihypertensive drug therapies. JAMA 1995; 274: 620-625.

39. Jick H, Derby L, Gurewich V, Vasllakis C: The risk of myocardial infarction in persons with uncomplicated essential hypertension associated with antihypertensive drug treatment. Pharmacotherapy 1996; 16: $321-326$.

40. Aursnes I, Litleskare I, Froyland H, Abdelnoor M: Association between various drugs used for hypertension and risk of acute myocardial infarction. Blood Pressure 1995; 4: 157-163.

41. Alderman $\mathrm{M}$, Cohen $\mathrm{H}$, Roque $\mathrm{R}$, Madhavan $\mathrm{S}$ : Long- and short-acting calcium antagonists differ with regard to cardiovascular outcomes in hypertensive patients. Lancet 1997; in press.

42. Mancia G, Van Zwieten P: How safe are calcium antagonists in hypertension and coronary heart disease? J Hypertens 1996; 14: 13-17.

43. Pahor M, Guralnik J, Corti M, Foley D, Carbonin P, Havlik R: Long-term survival and use of antihypertensive medications in older persons. $J A m$ Geriatr Soc 1995; 43: 1-7.

44. Koenig W, Lowel H, Lewis M, Hormann A: Longterm survival after myocardial infarction: relationship with thrombolysis and discharge medication. Results of the Augsburg Myocardial Infarction Follow-up Study 1985 to 1993. Eur Heart J 1996; 17: 1199-1206.

45. Braun S, Boyko V, Behar S, et al, on behalf of the Bezafibrate Infarction Prevention Study Participants: Calcium antagonists and mortality in patients with coronary artery disease: a cohort study of 11,575 patients. J Am Coll Cardiol 1996; 28: 7-11.

46. Abascal V, Larson M, Evans J, Poli K, Levy D: Calcium channel blocker use is not associated with increased mortality. Circulation 1996; 94: I-578.

47. Hamet P: Cancer and hypertension - an unresolved issue. Hypertension 1996; 28: 321-324.

48. Correale P, Tagliaferri P, Celio L, Genua G, Montagnani S, Bianco A: Verapamil upregulates sensitivity of human colon and breast cancer cells to LAKcytotoxicity in vitro. Eur J Cancer 1991; 27: 13931395.

49. Pahor M, Guralnik J, Salive M, Corti M, Carbonin P, Havlik R: Do calcium channel blockers increase the risk of cancer? Am J Hypertens 1996; 9: 695-699.

50. Pahor M, Guralnik J, Ferrucci L, et al: Calciumchannel blockade and incidence of cancer in aged populations. Lancet 1996; 348: 493-497.

51. Connor J, Sawczuk I, Benson M, et al: Calcium channel antagonists delay regression of androgen-dependent tissues and suppress gene activity associated with cell death. Prostate 1988; 13: 119-130.

52. Ray S, Kamendulis L, Gurule M, Yorkin R, Corcor- an G: $\mathrm{Ca}^{2+}$ antagonists inhibit DNA fragmentation and toxic cell death induced by acetaminophen. FASEB J 1993; 7: 453-463.

53. deBlois $\mathrm{D}$, Tea $\mathrm{B}$, Dam $\mathrm{T}$, Hamet $\mathrm{P}$ : Increased smooth muscle cell apoptosis during regression of vascular hypertrophy in the aorta of spontaneously hypertensive rats. Hypertension 1997; 29 (Suppl 2): in press.

54. Ekbom A, Adama H: Kalciumantagonister och cancer-en oversikt. Inf Lakemedelsverket 1996; 7: 3-5.

55. Hardell L, Fredrikson M, Axelson O: Case control study on colon cancer regarding previous diseases and drug intake. Int J Oncol 1996; 8: 439-444.

56. Pahor M, Guralnik J, Furberg C, Carbonin P, Havlik R: Risk of gastrointestinal haemorrhage with calcium antagonists in hypertensive persons over 67 years old. Lancet 1996; 347: 1061-1065.

57. Wagenknecht L, Furberg C, Hammon J, Legault C, Troost B: Surgical bleeding: unexpected effect of a calcium antagonist. $\mathrm{Br}$ Med J 1995; 310: 776-777.

58. Pilotto A, Leandro G, Franceschi M, Di Mario F, Valerio G: Antagonism to calcium antagonists. Lancet 1996; 347: 1761-1762.

59. Grodecki-De Franco P, Steinhubl S, et al: Calcium antagonist use and perioperative bleeding complications: an analysis of 5,157 patients. Circulation 1996; 94: I-476.

60. Hynynen M, Kuitunen A, Salmenpera M: Surgical bleeding and calcium antagonists [letter, comment]. Br Med J 1996; 312: 313.

61. The Danish Study Group on Verapamil in Myocardial Infarction: Verapamil in acute myocardial infarction. Eur Heart J 1984; 5: 516-528.

62. The Israeli SPRINT Study Group: Secondary prevention reinfarction Israeli nifedipine trial (SPRINT). A randomized intervention trial of nifedipine in patients with acute myocardial infarction. Eur Heart J 1988; 9: 354-364.

63. Multicenter Diltiazem Post-infarction Trial Research Group: The effect of diltiazem on mortality and reinfarction after myocardial infarction. $N$ Engl J Med 1988; 319: 385-392.

64. Psaty BM, Sevick MA: Antagonism to calcium antagonists. Lancet 1996; 347: 1761.

65. Robinson M, Teasdale G: Calcium antagonists in the management of subarachnoid haemorrhage. Cerebrovascular and Brain Metabolism Reviews. New York, Raven Press Ltd, 1990, pp 205-226.

66. Pickard JD, Murray GD, Illingworth R, et al: Effect of oral nimodipine on cerebral infarction and outcome after subarachnoid haemorrhage: British aneurysm nimodipine trial. $\mathrm{Br}$ Med $J$ 1989; 298: 636-642.

67. Mohr JP, Orgogozo JM, Harrison MJG, et al: Metaanalysis of oral nimodipine trials in acute ischemic stroke. Cerebrovasc Dis 1994; 4: 197-203.

68. Kaste M, Fogelholm R, Erila T, et al: A randomized, double-blind, placebo-controlled trial of nimodipine in acute ischemic hemispheric stroke. Stroke 1994; 25: $1348-1353$.

69. Takagawa Y, Shimamoto K, Masuda A, Iimura O: Survey of current drug therapy for elderly hypertension. Ther Res 1995; 16: 3822-3826.

70. Murros K, Furberg C: Calcium antagonists and extrapyramidal symptoms. Circulation 1996; 94: I-579.

71. Brown M: Calcium-channel blockers and cancer. Lancet 1996; 348: 1166.

72. Boston Collaborative Drug Surveillance Program: Reserpine and breast cancer. Lancet 1974; 2: 669671.

73. Armstrong B, Stevens N, Doll R: Retrospective study of the association between use of Rauwolfia de- 
rivatives and breast cancer. Lancet 1974; 2: 672-674.

74. Heinonen $\mathrm{O}$, Shapiro S, Tuominen L, Turunen M: Reserpine use in relation to breast cancer. Lancet 1974; 2: 675-677.

75. Horwitz R, Feinstein A: Exclusion bias and the false relationship of reserpine and breast cancer. Arch Intern Med 1985; 145: 1873-1875.

76. MRC Working Party: Medical Research Council trial of treatment of hypertension in older adults: principal results. Br Med J 1992; 304: 405-412.

77. SOLVD Investigators: Effect of enalapril on mortality and the development of heart failure in asymptomatic patients with reduced left ventricular ejection fractions. N Engl J Med 1992; 372: 685-691.
78. Walker A, Stampfer M: Observational studies of drug safety. Lancet 1996; 348: 489.

79. Buring J, Glynn R, Hennekens C: Calcium channel blockers and myocardial infarction. A hypothesis formulated but not yet tested. JAMA 1995; 274: 654655.

80. Easterbrook P, Berlin J, Gopalan R, Matthews D: Publication bias in clinical research. Lancet 1991; 337: 867-872.

81. Garg R, Yusuf S: Overview of randomized trials of angiotensin-converting enzyme inhibitors on mortality and morbidity in patients with heart failure. $J \mathrm{Am}$ Coll Cardiol 1995; 273: 1450-1456. 